\title{
Modeling Non-Stationary Processes of Diffusion of Solute Substances in the Near-Bottom Layer of Water Reservoirs: Variation of the Direction of Flows and Assessment of Admissible Biogenic Load
}

\author{
V.V. Kozlov * \\ Institute for System Dynamics and Control Theory, SB of RAS, Irkutsk, Russia
}

\begin{abstract}
The paper is devoted to mathematical modelling and numerical computations of a nonstationary free boundary problem. The model is based on processes of molecular diffusion of some products of chemical decomposition of a solid organic substance concentrated in bottom sediments. It takes into account non-stationary multi-component and multi-stage chemical decomposition of organic substances and the processes of sorption - desorption under aerobic and anaerobic conditions. Such a model allows one to obtain quantitative estimates of incoming solute organic substances of anthropogenic origin having different molecular weights from the bottom sediments into water and to study the influence of seasonal variations of the concentration of solute oxygen in the near-bottom water on the direction of exchange processes in the system "water-bottom".

Identification of parameters of the mathematical model with the use of experimental data and with the employment of a priori information of the model's structure is carried out. Comparison of the numerical simulations with experimental data is conducted to the end of verification of efficiency and plausibility of the proposed mathematical model of secondary pollution. It implies assessment of water quality on account of the processes of exchange in the system "water-bottom". The results of computations of non-stationary fluxes at the boundary "water-bottom" are analyzed. A model example is used to estimate the potentials of the biogenic load on the water reservoir.
\end{abstract}

Key words: mathematical modeling, bottom sediments, molecular diffusion, porous medium, secondary pollution, hydrodynamics, ecology, parametric identification

AMS subject classification: 92D40, 35K57, 76S05, 93A30, 93E12.

\footnotetext{
*E-mail: rozen@icc.ru
} 


\section{Introduction}

Almost the world-over sharpening of ecological problems related to water bodies makes essential the investigations of the possibility of obtaining the quantitative estimate of the values of the diffusion flow of solute organic substances from bottom sediments $[1-3,7,8,11,15-17,20]$. It is important to estimate the influence of the flow on the quality of water in the investigated water body.

Organic substances 1) undergo biochemical transformation in the water body, 2) are absorbed on the suspensions, 3) penetrate into bottom sediments. This leads to the decrease in the concentration of organic substances in the water. On the other hand, this leads to the result, when the bottom sediments become a long-term source of secondary pollution. In this case it is necessary to take into account that organic substances undergo biochemical transformation both in the water reservoir and in the bottom sediments $[2,3,11,15,16]$.

In the process of assessment and forecasting of processes of chemical decomposition of organic substances of bottom sediments it is necessary to take account of the value of concentration of solute oxygen in the near-bottom water. This value substantially influences both the rate of the processes of biochemical transformation of the substances and the content of these processes. This especially relates to the processes going on under the conditions of substantial deficit of the solute oxygen. In [1] one can find the estimate of the principal factors, which play the leading role in the variation of the intensity of oxygen consumption by bottom sediments, for the Mozhaisk water reservoir observed during a decade, in different seasons. In case of substantial variability of the process of consumption of oxygen by bottom sediments, we have noticed a positive dependence of this process on the content of oxygen in the near-bottom $(10 \mathrm{~cm})$ layer of water during all the seasons except for the periods of homotermia in spring and absence of stable temperature stratification in the reservoir in autumn. The correlation between the intensity of oxygen consumption with the content of organic substances in the upper layer $(0-2 \mathrm{~cm})$ of bottom sediments and consumption of oxygen in the near-bottom layer of water is also obvious. Furthermore, we have revealed a correlation between the intensity of consumption of oxygen by bottom sediments with the consumption of oxygen in the near-bottom layer of water. For example, in autumn, it is possible to observe a direct dependence, while in summer there is a reverse dependence, i.e., when there is no inflow of easily oxidizable organic substances to the bottom, the intensity of consumption of oxygen by bottom sediments is limited by intensive consumption of oxygen in the near-bottom layer of water.

\subsection{Physics-chemical characteristic of bottom sediments}

The weighted substance contained in the sewage of pulp-and-paper plants, which is discharged into water bodies, contains up to $35 \%$ of cellulose and up to $32 \%$ of lignin [9]. These hardly oxidizable organic substances undergo the process of sedimentation in bottom sediments in the form of black slimy mass, while changing the natural content of bottom sediments. The products of decomposition of above natural polymers are represented by toxic low-molecular organic substances, whose occurrence in the water reservoir leads to worsening its sanitary regime, reduction of the content of oxygen, violation of biochemical processes of self-purification. 
According to [9], decomposition of craft-lignin in sewage, which is registered via the chemical consumption of oxygen (CCO), is 50\% in 2 weeks and $90 \%$ in 40 weeks. It is considered that $50 \%$ decomposition of lignin with the stage of bleaching takes place during a week, and then CAO does not decrease during 40 weeks, after what disassimilation practically stops. Our special anxiety is caused by the presence of chlor-lignins in their content. There appear thousands of chlororganic substances, approximately 300 of which have been identified. Note, not less than $97 \%$ of the total chloride bound up with organic substances is represented by non-recognized mid-molecular and high-molecular organic substances, which are decomposed into chlorinated organic substances with lower molecular weight, many of which are chemically resistant toxic substances [18, 19].

According to [3] the organic substances of bottom sediments on the Baikal possess a relatively high concentration of easily hydrolyzable forms. Sediments of the upper layer of bottom sediments give 24 to $72 \%$ of organic carbon into the first hydrolyzate, 5 to $52 \%$ into the second one, and in the insoluble residue the content of carbon is from 10 to $70 \%$. It is noted that important factors, which determine the quantity of easily hydrolozable organic substance in bottom sediments, are the conditions of sedimentation. For the relatively high rates of sedimentation the organic substances undergo sedimentation in bottom sediments, but retain the capability of biochemical oxidation. We have shown the direct link between the mass content of organic carbon and the absolute quantity of its easily hydolizable forms.

To the end of assessment of an admissible biogenic load on the water reservoir it is necessary to know the process of spatial-temporal dynamics of the pollution sites on bottom of the water reservoir, into which industrial sewage waters are discharged. Our analysis has given evidence that the zone of high pollution has already formed. The total content of lignin-humus substances and cellulose in bottom sediments is $35-40 \%$. These zones of anthropogenic pollution have reached the size of some 20-40 sq. km during 30-50 years of work of the enterprises [9]. Naturally, hardly oxidizable organic substances are present in the water reservoir also as a natural component. Even in oligotrophic reservoirs its content may be several milligrams in $\mathrm{dm}^{3}$ [3]. So, getting CAO into a pretty large water reservoir from sewage waters may be not very substantial in its percentage. One has to remember that the content of the complex of substances, which are determined from CAO, includes low-molecular substances, which are foreign for all ecosystems and are hard to remove. The difficulties of organization of valuable natural observations of such processes for the purpose of getting the estimate of influences on the ecosystem have made us to use mathematical modeling $[2,7,8,11]$.

\subsection{Problem statement}

Bottom sediments represent a natural porous medium saturated with water solute of different organic and mineral substances, which is formed in the water basin by sequential adding of a large number of particles falling onto the bottom, which have different dimensions and content. This environment is bounded from below by the fundamental bed of the water reservoir, and from above - by the interface with the water layer, from now on - the boundary "water-bottom". For the purpose of definiteness, let us consider the boundary "water-bottom" coinciding with the boundary of diffusion boundary layer [12]. For large Pekle numbers, the characteristic speed of the concen- 
tration variation in the boundary layer in the direction transversal to the flow is essentially greater than in the longitudinal direction.

Since exact quantitative description of the structure of real porous medium and of the diffusion transfer in it is related to substantial difficulties, in case of a macroscopic approach it is generally considered as some homogenized continuous medium. Effective parameters of this medium are chosen to provide equality of diffusion flows with the real porous medium under equivalent conditions.

Next, we assume that, except for formation of volatile substances under anaerobic conditions, the rest of the chemical reactions going on under the two considered conditions (aerobic and anaerobic) are similar. Decomposition of the mass of organic substance under anaerobic conditions is considered as the first-order reaction. This relatively simple model of biochemical reaction rather exactly reflects the process of integration in bottom sediments [16]. This model of integration for the initial organic substances, both easily oxidized and hardly oxidizable ones, is used in [20]; a kinetic model of regeneration of inorganic compounds of biogenic elements under anaerobic conditions is also described in [20].

Unlike that, under anaerobic conditions, there may take place reactions of two types. Kinetics of reactions, which belong to the first type, is described by the Mihaelis-Menten equation, which considers variation of the biomass (the Monot function for the substrate concentration). Second type processes are described by the first-order reactions which characterize variation of the total content of volatile substances. In the presence of a sufficiently large concentration of the substrate (initial organic substance) and when the process of integration is assessed under anaerobic conditions we shall use a simpler first-order kinetic equation, which takes account of variation of biomass (instead of the Mihaelis-Menten equation). Under anaerobic conditions, we assume that the rate of disassimilation of the solute organic substance is proportional to the content of the solute oxygen [10].

Description of the process of decomposition of the organic substances. Consider disassimilation of the organic substance in bottom sediments, following the scheme proposed in [11], as going on at the same time but independently of multistage hydrolysis (decomposition) of some of its "kinetic groups", resulting in formation of inorganic final products. Suppose disassimilation is satisfactorily described by the first-order kinetics. The initial substance of the bottom sediments and products on each stage of disassimilation will be denoted by $P_{i j}$, where $i=0,1, \ldots, n_{j}$ is the number of the substance (product) of the $j$-th group, and $j=0,1, \ldots, m$ the number of the group. In the multistage process, decomposition of the $(i-1)$-st intermediate product adds to the quantity of the $i$-th product.

Hence the general scheme of decomposition of the organic substance in bottom sediments can be represented as follows:

$$
P_{0 j} \rightarrow P_{1 j} \rightarrow P_{2 j} \rightarrow \ldots \rightarrow P_{\left(n_{j}-1\right) j} \rightarrow P_{\left(n_{j}\right) j}
$$

where: $P_{0 j}$ - initial organic substance of the $j$-th group, $P_{\left(n_{j}\right) j}$ - final inorganic product of the given group. All the intermediate products form a solute organic substance. Then $\sum_{j=1}^{m} P_{0 j}$ 
characterize the initial organic substance of bottom sediments, and the value $\sum_{i=1}^{n_{j}-1} \sum_{j=1}^{m} P_{i j}$ is the sum of solute organic products in porous solutes.

It is supposed that substantial variations of the value and the direction of the diffusion flow of solute organic substance through the boundary "water-bottom" take place only during transient processes. These transient processes depend on the conditions of the environment external with respect to the system "near-bottom water - bottom sediments". The external conditions determine the variations of solute oxygen in the reservoir's water and the concentration of solute and suspended organic substances in the "near-bottom layer" of the water. The sorption equilibrium under aerobic and anaerobic conditions is established quickly, and, at each time moment, the current concentration of solute organic substances is practically equal to the equilibrium one because we assume that the time of sorption-desorption is negligibly small with respect to the time, which determines changes under the external conditions. The coefficient of convertible sorption is satisfactorily described by the linear Henry law and has different values in the upper oxidized and lower reduced layers of bottom sediments, and the coordinate of the boundary between the layers is a function of time.

\section{Mathematical model and the method of investigation}

A local moving coordinate system attached to the boundary "water-bottom" is introduced. The origin coincides with the given boundary, the positive axis $0 \mathrm{z}$ goes downwards, into the layer of sediments. Since the change of the concentration per unit of length along the vertical line in bottom sediments is much larger than variations of the value of concentration in other directions, the diffusion process will be considered as a one-dimensional process. In course of sedimentation (with the sedimentation rate $V_{o c}$ ) the coordinate system moves upwards parallel to itself with the velocity $V_{o c}$.

\subsection{Equations of molecular diffusion in a porous medium}

Generalizing the results of $[7,8,11]$ and taking into account mass transfer due to molecular diffusion, we can write micro-kinetics equations describing the processes in bottom sediments in the form:

$$
\begin{gathered}
\frac{\partial\left[G_{i j}(z, t) C_{i j}(z, t)\right]}{\partial t}=\frac{\partial}{\partial z}\left[D_{i j}^{e f}(z, t) \frac{\partial C_{i j}(z, t)}{\partial z}\right]-\frac{\partial}{\partial z}\left[G_{i j}(z, t) V_{o c}(z, t) C_{i j}(z, t)\right]+f_{i j}(z, t) \\
i=0,1, \ldots, n_{j}, \quad j=0,1, \ldots, m, \quad z \in[0, L], \quad t \in[0, T]
\end{gathered}
$$


Here $C_{p}(z, t)=\sum_{i=1}^{n_{j}-1} \sum_{j=1}^{m} C_{i j}(z, t)$ is the sum of solute organic products in porous solutes, $C_{0}=$ $\sum_{j=1}^{m} C_{0 j}$ is the initial mass of organic substances of bottom sediments. $C_{i j}(z, t)$ is the concentration of the organic substance (solid and solute) in porous sediments, which is expressed in units of chemical consumption of oxygen $\left(\mathrm{mgO}_{2} / \mathrm{dm}_{\text {general }}^{3}\right)$ with respect to the unit of volume of water saturated porous sediment. The effective coefficient of molecular diffusion is $D_{i j}^{e f}(z)=D_{i j}^{0} p^{m}(z)$, where $1,3<m<3$. The formula for the effective coefficient of molecular diffusion includes the porosity $p(z)$, and the coefficient of molecular diffusion $D_{i j}^{0}$ of the substance solute in water in case of infinite dilution $[11,14,17]$. In this work, it is supposed that $m=2$.

Since the initial solid organic substance does not diffuse, $D_{0 j}^{0}=0$ for all kinetic groups. When the rate of the sorption process is sufficiently high, it is supposed that the equilibrium between the liquid and solid phases is established instantaneously and it is described by a linear isotherm line. The process of equilibrium sorption is described by the function $G_{i j}(z, t)=1+K_{i j}^{a}(z, t)$, where

$$
K_{i j}^{a}(z, t)= \begin{cases}K_{i j}^{a 1}, & 0 \leq z \leq z^{*}(t) \\ K_{i j}^{a 2}, & z^{*}(t) \leq z \leq L .\end{cases}
$$

Here $z^{*}(t)$ is the thickness of the oxidized layer of the bottom sediments.

In the general case, the functions in the right-hand side of the equation have the form

$$
f_{i j}(z, t)=-k_{i j}(z, t) C_{i j}(z, t)+k_{(i-1) j}(z, t) C_{(i-1) j}(z, t)+f_{i j}^{0}(z, t)
$$

and describe the process of chemical decomposition of the organic substance, as well as mass production, chemical reactions, physical-chemical and biological processes, which involve variations of concentration with time $[9,11]$. The rate of chemical decomposition of the substance $k_{i j}(z, t)$ (1/hour) depends on the content of oxygen solute in the bottom sediments. It has different values in the aerobic and anaerobic domains of the bottom sediments and of the "near-bottom water". In this case, constants of rates of multi-stage and multi-component decomposition of substances $k_{i j}(z, t)$ depending on the content of oxygen, that is on position of the boundary of the oxidized layer of sediments in bottom sediment. They can be written as follows:

$$
\begin{gathered}
k_{i j}(z, t)=\left\{\begin{array}{c}
k_{i j}^{p} C_{k}(z, t), \quad 0 \leq z \leq z^{*}(t), \quad i=1,2, \ldots, n_{j}-1, \quad j=0,1, \ldots, m, \\
k_{i j}^{p} \beta_{i j}, \quad z^{*}(t) \leq z \leq L,
\end{array}\right. \\
k_{0 j}(z, t)=\left\{\begin{array}{c}
k_{j}^{0}, \quad 0 \leq z \leq z^{*}(t), \\
k_{j}^{0} \beta_{j}^{0}, \quad z^{*}(t) \leq z \leq L, \quad j=0,1, \ldots, m,
\end{array}\right.
\end{gathered}
$$

where $C_{\kappa}(z, t)$ is the concentration of oxygen solute in bottom sediments, and the dimensionless coefficients $\beta_{i j}, \quad \beta_{j}^{0}$ take account of the difference in rates of processes under aerobic and anaerobic conditions. We note that under real conditions existing for the initial substance of bottom sediments of fresh-water reservoirs there exists an approximate estimate $\beta_{j}^{0} \approx 0.5$ [11]. For all finite products of chemical decomposition, the rate of decomposition is $k_{\left(n_{j}\right) j}(z, t)=0$. As far as the initial organic substance is concerned, there are no positive sources in bottom sediments, and, consequently, $C_{0 j}(z, t)=0$ for all kinetic groups. 
The total flux for each product in bottom sediments is given by the formula

$$
F_{i j}^{p}(z, t)=-\left[D_{i j}^{e f}(z) \frac{\partial C_{i j}(z, t)}{\partial z}\right]+\left[G_{i j}(z, t) V_{o c}(z, t) C_{i j}(z, t)\right] .
$$

The total flux of all solute organic products of disassimilation can be written as

$$
F^{p}(z, t)=\sum_{i=1}^{n_{j}-1} \sum_{j=1}^{m} F_{i j}^{p}(z, t) .
$$

The expression for the total flux through the surface of bottom sediments for each product is $F_{i j}^{p}(t)=\left.F_{i j}^{p}(z, t)\right|_{z=0}$, at the boundary "water-bottom" defined above.

Initial and boundary conditions. 1. The initial vertical distribution of the concentration of solute organic substance in the sediment is $\left.C_{i j}(z, t)\right|_{t=0}=\Psi_{i j}(z)$. The function $\Psi_{i j}(z)$ is found from the solution of the problem of parametric identification with the use of experimental data for the stationary mathematical model [8] or by the method of relaxation for numerical solution of system (2.1).

2. At the lower boundary of the bed of the water reservoir of the anthropogenic layer of bottom sediments with the coordinate $z=L$ the concentration gradient is zero:

$$
\left.\frac{\partial C_{i j}(z, t)}{\partial z}\right|_{z=L}=0 .
$$

3. The boundary condition at the boundary "water-bottom" is $\left.C_{i j}(z, t)\right|_{z=0}=C_{i j}^{*}(t)$. Here the concentration $C_{i j}^{*}(t)$ are the given functions of time, which define variations of the concentration of substance at the boundary "water-bottom".

\subsection{Consumption of oxygen by bottom sediments}

Unlike solute organic products, oxygen has no positive sources in the layer of bottom sediments. It comes into sediments through the boundary "water-bottom". Variations of the content of oxygen in water of the near-bottom layer of a shallow water reservoir provoke the related variations of oxygen concentration in the porous solutes of the upper layer of the sediments. This provokes the change in time needed for deep penetration of oxygen into bottom sediments and in the conditions of reduction-oxidation processes there, and is reflected in the course of the total complex of physicalchemical processes in the upper layer of the sediments.

Diffusion of oxygen $C_{\kappa}(z, t)$ into bottom sediments of shallow water reservoir, which is accompanied by its consumption with the rate proportional to its concentration, can be written in the following form $[7,11]$ :

$$
\frac{\partial C_{\kappa}(z, t)}{\partial t}=\frac{\partial}{\partial z}\left[D_{k}^{e f}(z, t) \frac{\partial C_{\kappa}(z, t)}{\partial z}\right]-\frac{\partial}{\partial z}\left[V_{o c}(z, t) C_{\kappa}(z, t)\right]+\Phi_{\kappa}(z, t) .
$$


Function $\Phi_{\kappa}(z, t)$ in the right-hand side of (2.2) describes the kinetics of reaction of organic substances of bottom sediments with oxygen and, according to the mass action law, writes as follows $[9,11]$ :

$$
\Phi_{k}(z, t)=-\sum_{j=1}^{m}\left(\sum_{i=1}^{n_{j}-1} k_{i j}^{p} C_{i j}(z, t)+k_{j}^{k} C_{0 j}(z, t)\right) C_{k}(z, t),
$$

where $k_{j}^{k}$ and $k_{i j}^{p}\left(d m_{\text {general }}^{3} /\left(m g O_{2} \cdot 24\right.\right.$ hours $\left.)\right)$ are the rate constants for the initial and solute organic substances of bottom sediments for anaerobic decomposition; the reaction is irreversible.

The density of the total flux of solute oxygen in bottom sediments is computed by the formula: $F_{k}^{p}(z, t)=-\left[D_{k}^{e f}(z) \frac{\partial C_{k}(z, t)}{\partial z}\right]+\left[V_{o c}(z, t) C_{k}(z, t)\right]$.

\section{Initial and boundary conditions.}

1. At the initial time moment, the vertical distribution of concentration of solute oxygen in the bottom sediments $\left.C_{\kappa}(z, t)\right|_{t=0}=\varphi(z)$ is given.

2. At the lower boundary of the anthropogenic layer of bottom sediments with the coordinate $z=L$ the concentration gradient is zero, $\left.\frac{\partial C_{\kappa}(z, t)}{\partial z}\right|_{z=L}=0$.

3. At the boundary "water-bottom" $\left.C_{\kappa}(z, t)\right|_{z=0} ^{z=L}=C_{k}^{*}(t)$, where the concentration of the solute oxygen in the near-bottom water $C_{k}^{*}(t)$ can be found like $C_{i j}^{*}(t)$ or be of a given value.

\subsection{Dynamics of the surface oxidized layer}

For estimating the dynamics of the surface of the oxidized layer of sediments let us use the following assumptions. On the basis of experimental investigations, it has been shown [11] that under the boundary of the oxidized layer the content of oxygen solute in the porous solute is small, it is about $\tilde{C}_{\text {min }}=0,5 \mathrm{mgO}_{2} / \mathrm{dm}_{\text {porous }}^{3}$. In this connection, we shall consider the concentration of oxygen at the lower boundary of the oxidized layer to be practically constant. The time change of the position of the level line in the sediment corresponding to the given concentration describes the dynamics of thickness of the surface of the oxidized layer, which is conditioned by variations of the oxygen content in the near-bottom water $H(z, t)=C_{\kappa}(z, t)-p(z) \tilde{C}_{\min }=0$.

The velocity of the lower boundary of the oxidized layer is given by the equation:

$$
\frac{d z^{*}}{d t}=-\left.\frac{\frac{\partial C_{k}}{\partial t}}{\frac{\partial C_{k}}{\partial z}-\tilde{C}_{\min } \frac{\partial p}{\partial z}}\right|_{z=z^{*}} .
$$

The initial position $\left.z^{*}(t)\right|_{t=0}=z_{0}^{*}$ is determined by the initial vertical distribution of solute oxygen $\varphi(z)$.

\subsection{Numerical method}

In the general case, we obtain a boundary-value problem (2.1), (2.2) for the equations of parabolic type with the boundary conditions imposed on moving boundaries (2.3), the boundary "waterbottom" and the boundary of the oxidized layer of bottom sediments. For discretization with 
respect to the spatial variable we use an upwind finite difference scheme for the convective terms and central differences for the diffusion component [4].

The principle of frozen coefficients [4] allows one to separately solve the problem for the distribution of solute organic substances and then to determine the location of the moving boundary. We consider iterative method of Richardson[4]. Suppose the time segment $\left[T_{0}, T_{1}\right]$ is decomposed into intervals $\left(t_{n-1}, t_{n}\right)$, within which the system's coefficients satisfy the conditions of stability and convergence. As the basis of numerical implementation of the problem on the time interval $\tau=t_{n}-t_{n-1}$ we take the explicit scheme of local iterations LI-M [5, 6]. Results of computations conducted for test examples have shown that this scheme for parabolic equations of molecular diffusion with constant coefficients is stable [8].

\section{Results of computations for bottom sediments}

Consider a simplified model example which reflects the real situation. We study one-component process with respect to lignin substances which form over $61 \%$ of the initial composition of bottom sediments. The process has two stages of disassimilation. Consider the initial data for parametric identification of decomposition rates.

At the first stage, there are organic substances with the diffusion coefficient $D_{1}^{0}=2.95$. $10^{-6} \mathrm{sm}^{2} / \mathrm{sec}\left(25^{\circ} \mathrm{C}\right)$. We emphasize that this is an approximate value used for test computations. For comparison, for high-molecular weight mioglobin diffusion coefficient is $D_{m i o}^{0}=$ $4.4 \cdot 10^{-7} \mathrm{sm}^{2} / \sec \left(37^{\circ} \mathrm{C}\right)$ [10]. At the second stage, low-molecular organic substances are formed. Their molecular mass is close to the ion of hydrogen carbonate. It has the coefficient of molecular diffusion $D_{2}^{0}=11.8 \cdot 10^{-6} \mathrm{sm}^{2} / \sec \left(25^{\circ} \mathrm{C}\right)$ [11]. The coefficient of molecular diffusion for oxygen is accepted equal to $D_{k}^{0}=23.0 \cdot 10^{-6} \mathrm{sm}^{2} / \sec \left(25^{\circ} \mathrm{C}\right)$ [17].

Variation of oxygen concentration in the near-bottom layer is given by the equation $C_{k}^{*}(t)=$ $9,02 \cdot(1+\cos (\pi t / 30)) \mathrm{mg} / \mathrm{dm}^{3}$. The values larger than the amplitude of variations are cut off.

The constant of sorption in the upper oxidized layer is two times as large as the value of sorption in the lower anaerobic layer for all the products of chemical decomposition

$$
K_{i}^{a}(z, t)=\left\{\begin{array}{cc}
10, & 0 \leq z \leq z^{*}(t) \\
5, & z^{*}(t) \leq z \leq L
\end{array} .\right.
$$

The rate of chemical decomposition of the initial organic substance is different under anaerobic and aerobic conditions. It differs approximately two times. The sedimentation rate, estimated through the thickness of the layer of bottom sediments, is approximately $15 \mathrm{~mm}$ per year.

The rate constants of the two-stage process were determined from the results of parametric identification, for the solid organic substance $k^{0}=0,000421 / 24 h$, for the solute organic products of anaerobic decomposition $k_{1}^{p}=0,012 \mathrm{dm}^{3} / \mathrm{mg} \cdot 24 \mathrm{~h}, k_{2}^{p}=0,024 \mathrm{dm}^{3} / \mathrm{mg} \cdot 24 \mathrm{~h}$, the coefficients of anaerobic decomposition $\beta_{1}=\beta_{2}=7,5 \mathrm{mg} / \mathrm{dm}^{3}$. Fig. 1 shows a sufficiently good correspondence of the numerical simulations and experimental data within the limits of measurement errors of $10-15 \%$.

The results of computations given in Fig. 2 show that during transient processes related to variation of oxygen concentration in the near-bottom layer of water, the value of the total flow of 

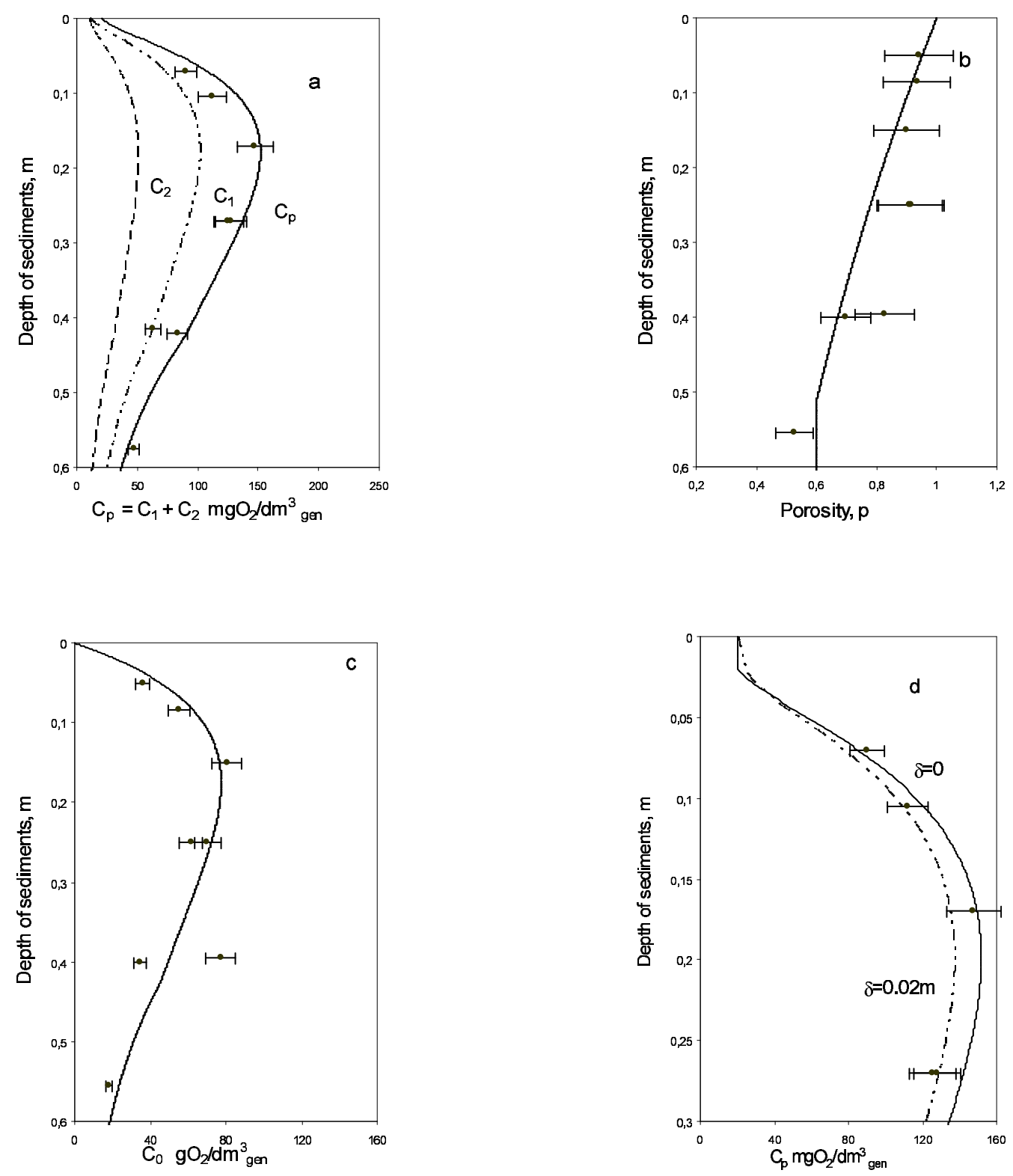

Figure 1: Comparison of numerical simulations with the experimental data for a a two-stage process of chemical decomposition. Relative error is from 10 to $15 \%$. Layers of bottom sediments of approximately equal thickness $\sim 60-65 \mathrm{~cm}$, the distance between them being $\sim 1200 \mathrm{~m}$ ) samples of ground columns taken in a bay of the reservoir [8]. Diagram (d) numerical and experimental results for "the near-bottom layer of water" of thickness of $0.02 \mathrm{~m}$ (dotted line). The figure (a) shows the concentrations of the first (dotted curve) and the second (dashed curve) stages of organic disassimilation, and shows theirs sum (solid curve). 
solute organic substances from bottom sediments substantially growth. The first maximum, which shows a substantial increase of the flow, is related to occurrence of anaerobic conditions. The second maximum is smaller, it corresponds to diffusion of oxygen into the near-bottom layer.

In this case, the total value of the secondary pollution of water increases. Consumption of oxygen by bottom sediments does not exceed $100 \mathrm{mg}$ of solute oxygen per a square meter during 24 hours.

Fig. 3 shows the damping influence of "the near-bottom layer of water" (of thickness only $0.02 \mathrm{~m}$ ) on the bottom sediments: the source of secondary pollution. The value of maximum flow of solute organic substances decreases approximately by 3 times and, consequently, the biogenic load on the water reservoir is reduced. Consumption of oxygen by bottom sediments is reduced by approximately 2 times. The situation is more explicitly shown in Fig.1d, where it is clearly seen that the behavior of the concentration curves in the pore-filling solute and in the near-bottom layer of water differs inside this layer. The gradient of concentration decreases.

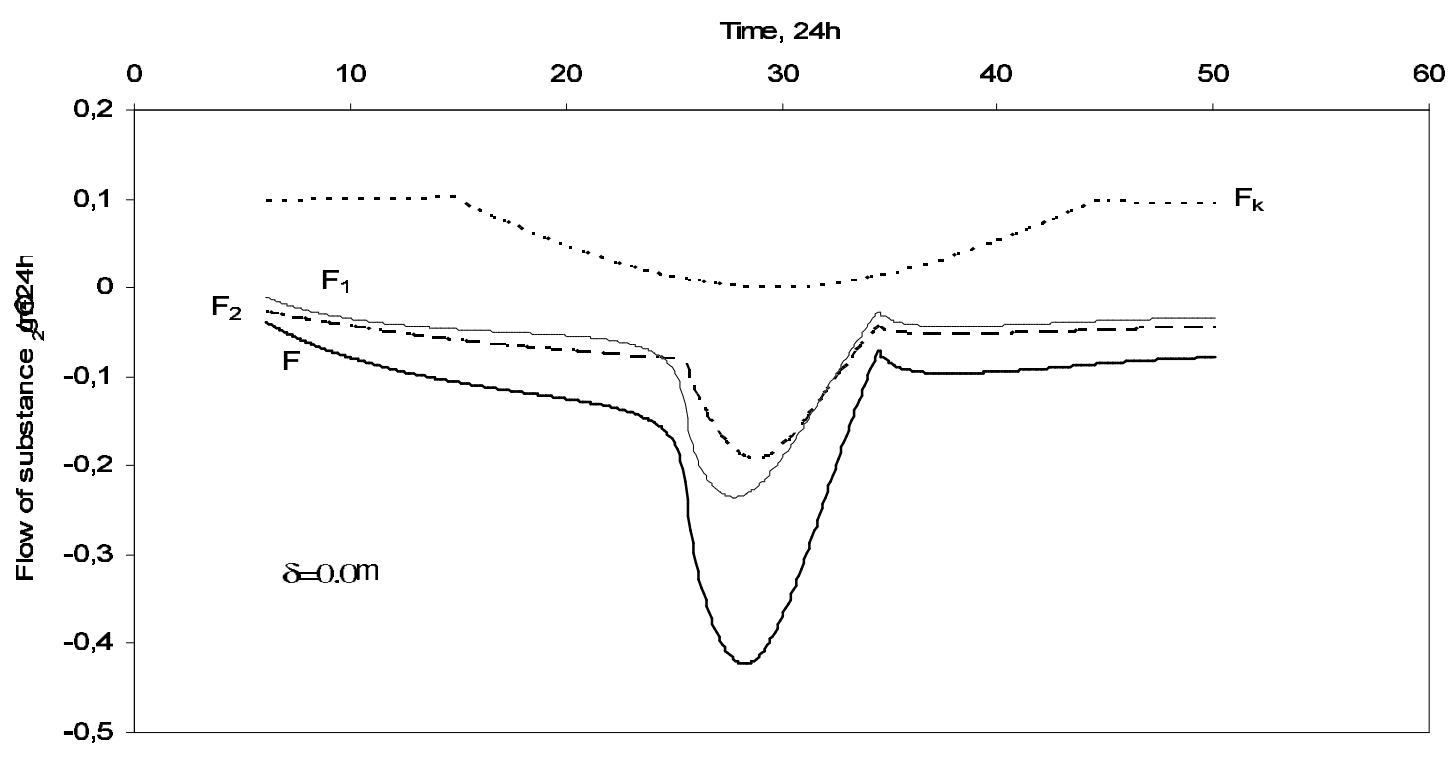

Figure 2: The value and the direction of diffusion flow of solute organic substances (thick solid curve) and oxygen (dotted curve) at the boundary of bottom sediments, the thickness of the nearbottom layer being zero. The computations have been conducted for the example of two-stage disassimilation of the organic substance in bottom sediments. There is flow of substance for the first (thin solid curve) and second (dashed curve) stages of organic disassimilation. 


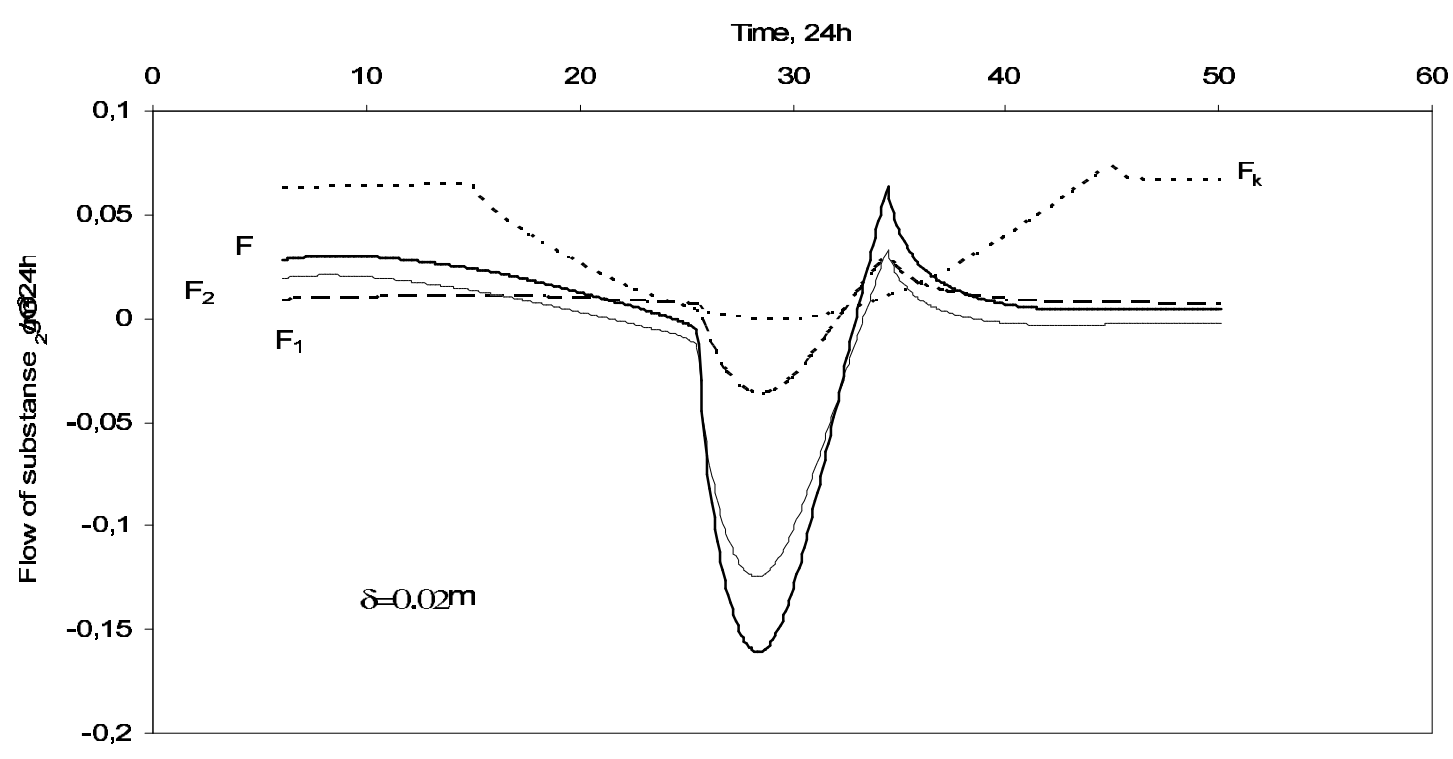

Figure 3: The value and the direction of diffusion flows of solute organic substances (thick solid curve) and oxygen (dotted curve) at the boundary of the "near-bottom layer" with the thickness $0.02 \mathrm{~m}$. The computations have been conducted for the example of two-stage disassimilation of the organic substance in bottom sediments. There is flow of substance for the first (thin solid curve) and second (dashed curve) stages of organic disassimilation. 


\section{Conclusion}

In this work, we study macro-kinetic processes in bottom sediments of water reservoirs with a water-saturated porous medium of variable porosity with respect to the depth of sediments. We describe consumption of oxygen by water sediments, multistage decomposition of organic substances, distribution of the products of disassimilation in pore-filling solutes. Instead of the conception of the barrier oxidized layer of bottom sediments, which prevents free diffusion exchange of solute substances with the near-bottom water, we have considered the concept of transient processes in sediments of water reservoirs with temporary lack of oxygen. Formation of diffusion flows of solute substances, which arise in sediments of a shallow water reservoir under quasiperiodic changes of conditions at the boundary "water-bottom" and in the near-bottom layer of water, has been investigated and described. To obtain a more plausible estimate of the secondary pollution related to transient processes it is necessary to estimate the whole complex of external influences on the water reservoir, while employing more advanced techniques of sampling of nearbottom water and bottom sediments would allow one to avoid disrupting the sample structure.

The results of computations give evidence that stationary models allow parametric identification of the parameters of the mathematical model on the basis of experimental data. Meanwhile, within the frames of non-stationary models, which describe transient processes, it is necessary to find an optimal combination of external impacts such that it would allow one to plausibly estimate the value of maximum flow of solute organic substances of anthropogenic origin from bottom sediments and assess the maximum possible biogenic load on the water reservoir. In course of further investigations of material exchange inside the system "water-bottom" it is necessary to conduct quantitative assessment of influence of both stirring-up and activity of zoobenthos organisms, as well as assessment of influence of their density on the flow of solute substances, while considering these as a part of the complex of external influence on the processes in bottom sediments.

\section{References}

[1] V.F. Brekhovskikh, G.N. Vishnevskaya, N.A. Gashkina et al. On seasonal change of priority factors, which determine the intensity of consumption of oxygen by grounds of the valley-type water reservoir. Vodniye Resursy, 30 (2003), No. 1, 61-66 (in Russian).

[2] O.F. Vasilyev, A.F. Voevodin. Mathematical modeling of water quality in systems of open river beds. Dynamica Sploshnoy Sredy. Novosibirsk, 22 (1975), 73-88 (in Russian).

[3] L.A. Vykhristyuk. Organic substance of bottom sediments of Lake Baikal. Novosibirsk. Nauka, 1980 (in Russian).

[4] S.K. Godunov, V.S. Ryabenky. Difference schemes. Moscow. Nauka, 1973 (in Russian).

[5] V.T. Zhukov. Explicitly iterative schemes for parabolic equations. Voprosy Atomnoy Nauky i Techniky. Mathematical Modeling of Physical Processes, 4 (1993), 40-46 (in Russian). 
[6] F.S. Zaytsev, D.P. Kostomarov, I.I. Kurbet. Application of explicit iterative schemes for solving kinetic problems. Mathematical Modeling, 16 (2004), No. 3, 13-21 (in Russian).

[7] V.V. Kozlov. Elaboration and identification of a non-stationary mathematical model of distribution of polluting substances in a water body. Proc. Intern. Conf. "Computational and Information Technologies in Science, Engineering and Education", Pavlodar, I (2006), 641649 (in Russian).

[8] V.V. Kozlov. Elaboration and identification of a non-stationary mathematical model of distribution of polluting substances in a water object. Irkutsk, 2006. (Preprint of SB RAS. ISDCT. No. 1) (in Russian).

[9] A.V. Bogdanov, G.D. Rusetskaya, A.P. Mironov, M.A. Ivanova. Complex process of decomposition of the waste of pulp and paper plants. Irkutsk Polytechnic Publishing, Irkutsk, 2000 (in Russian).

[10] G. Marry. Nonlinear differential equations in biology. Lectures on Models. Mir, Moscow, 1983.

[11] I.B. Mizandrontsev. Chemical processes in bottom sediments of water reservoirs. Nauka, Novosibirsk, 1990 (in Russian).

[12] L.G. Loytsyansky. Mechanics of liquid and gas. Nauka, Moscow, 1987 (in Russian).

[13] P. Rouch. Computational hydrodynamics. Mir, Moscow, 1980.

[14] Yu.I. Chizmadzhayev, V.S. Markin, M.P. Tarasevich, Yu.G. Chirkov. Macrokinetics of processes in porous media (Fuel elements). Nauka, Moscow, 1971 (in Russian).

[15] D.R. Aguilera, P. Jourabchi, C. Spiteri, and P. Regnier. A knowledge-based reactive transport approach for the simulation of biogeochemical dynamics in earth systems. Geochemistry, Geophysics and Geosystems, 6 (2005).

[16] R.A. Berner. Early diagenesis: a theoretical approach. Princeton University Press, Princeton, 1980.

[17] A. Lerman et al. Geochemical processes: water and sediment environments. Wiley Interscience, New York, 1979.

[18] W.D. Murray and M. Richardson. Development of biological and process, technologies for the reduction and degradation of pulp mill wastes that pose a threat to human health. Critical Reviews in Environmental Science and Tachnology, 23 (1993). 1157-1194.

[19] J. Paasivirta. Chemical ecotoxicology. Lewis Publishers, Chelsea, Michigan, 1991.

[20] P. Regnier, P. Jourabchi, and C.P. Slomp. Reactive-transport modeling as a technique for understanding coupled biogeochemical processes in surface and subsurface environments. Netherlands Journal of Geosciences, 82 (2003), 5-18. 